\title{
Estimación de Parámetros del Suelo de dos Capas usando el Algoritmo de Optimización de Mapeo Media-Varianza
}

\author{
Walter M. Villa-Acevedo, Johnatan M. Rodríguez-Serna y Juan D. Saldarriaga-Loaiza \\ Grupo de Investigación GIMEL, Departamento de Ingeniería Eléctrica, Facultad de Ingeniería, Universidad \\ de Antioquia, Calle 70 No. 52-21, Medellín - Colombia (e-mail: walter.villa@udea.edu.co; \\ jmauricio.rodriguez@udea.edu.co; juand.saldarriaga@udea.edu.co)
}

Recibido Abr. 25, 2018; Aceptado Jun. 28, 2018; Versión final Ago. 1, 2018, Publicado Feb. 2019

\begin{abstract}
Resumen
En este artículo se analiza la aplicación de la técnica de optimización de mapeo media-varianza en el problema de estimación de los parámetros del modelo de suelo de dos capas horizontales. El problema consiste en determinar los parámetros del modelo del suelo a partir de las mediciones experimentales de resistividad aparente obtenidas con el método de Wenner, minimizando el error medio cuadrático entre valores de las curvas de resistividad experimental y teórica, calculadas con expresiones matemáticas y parámetros del suelo obtenidos por la técnica de optimización de mapeo media-varianza. Se realizaron varias pruebas con medidas de resistividad que corresponden a diferentes tipos de suelos, comparando los resultados obtenidos con los reportados en la literatura técnica. En conclusión, de acuerdo a los resultados, se encontró que el desempeño de la técnica de optimización de mapeo media-varianza es superior a las otras técnicas de optimización analizadas.
\end{abstract}

\section{Estimation of Two-Layer Soil Parameters using Mean-Variance Mapping Optimization Algorithm}

\begin{abstract}
This paper presents an analysis of applying the optimization of mean-variance mapping technique in the problem of estimating the parameters of the soil model of two horizontal layers. This problem consists of determining the parameters of the soil model from experimental measurements of apparent resistivity obtained with Wenner method, minimizing the mean square error between values of the experimental and theoretical resistivity curves, which are calculated with mathematical expressions and soil parameters acquired through the optimization of mean-variance mapping technique. Several tests were carried out with resistivity measurements that correspond to different soil types, the results were contrasted with those reported in the technical literature and metaheuristics implemented. In conclusion, according to the results, the performance of the optimization of mean-variance mapping technique was found to be superior than other analyzed optimization techniques.
\end{abstract}




\section{INTRODUCCIÓN}

Los sistemas de puesta a tierra juegan un papel importante en el esquema de protecciones de cualquier instalación eléctrica. El valor de la resistencia de puesta a tierra debe permitir que las protecciones eléctricas actúen de manera adecuada y, además, debe garantizarse que las personas en los alrededores no estén sometidas a tensiones de contacto o de paso que superen los umbrales de soportabilidad (IEEE, 2015). La resistencia de puesta a tierra de un sistema depende de parámetros como la resistividad y el tipo de suelo, material y geometría de los conductores enterrados, pero es la resistividad eléctrica del suelo la que mayor influencia tiene no sólo en el valor de la resistencia de puesta a tierra, sino en el comportamiento mismo del sistema durante las fallas. Y es que este parámetro no sólo depende del tipo de suelo, sino que además depende de parámetros ambientales como la temperatura, la humedad y también de la estructura del suelo mismo (Moreno et al.,2007).

Para diseñar un sistema de puesta a tierra que sea técnicamente adecuado, se requiere conocer, entre otros, la resistividad eléctrica del suelo y la configuración geométrica del electrodo (IEEE, 2015). La resistividad eléctrica del suelo puede obtenerse a través de técnicas de prospección geoeléctrica como el método de Wenner (IEEE, 2012). La interpretación de los datos obtenidos a partir de las mediciones realizadas permite generar un modelo de suelo que sea representativo del suelo físico real que se tiene en el sitio, lo que puede llevar a modelos de suelo muy diversos que pueden ser desde un suelo homogéneo compuesto por una sola capa uniforme hasta modelos compuestos por dos capas o más, para los cuales métodos de diseño simplificado como el propuesto por la norma IEEE 80 (IEEE, 2015) pierden validez. Así, modelar el suelo en forma apropiada permite determinar el método de solución más adecuado para diseñar un sistema de puesta a tierra específico que cumpla con los requerimientos establecidos y de ahí la importancia de establecer un procedimiento preciso que permita la obtención del modelo de suelo a partir de las mediciones de resistividad en sitio. Un modelo del suelo aceptado considera capas horizontales con resistividad homogénea y la profundidad respectiva de cada capa (Tagg, 1964).

Se han desarrollado expresiones matemáticas que relacionan la resistividad aparente calculada con los parámetros del modelo de suelo de dos capas horizontales y la distancia entre los electrodos de medición (Sunde, 1949; Tagg, 1964). Los valores estimados de la resistividad aparente teórica se comparan con los valores medidos de resistividad; esta comparación se propone como un problema de ajuste de mínimos cuadrados entre las curvas de resistividad aparente. Comúnmente, se ha planteado la estimación de los parámetros del modelo del suelo como un problema de optimización no lineal con restricciones, se han usado herramientas de optimización clásica ( Alamo, 1991, 1993; Seedher y Arora, 1992) y técnicas metaheurísticas (Gonos y Stathopulos, 2005). La estimación con herramientas de optimización clásica presenta inconvenientes en la convergencia en los algoritmos debido a la presencia de mínimos locales y a la sensibilidad al punto de inicio del algoritmo. Las técnicas metaheurísticas no presentan los inconvenientes anteriores, pero no se puede garantizar el óptimo global ni que siempre se obtenga el mismo resultado.

En relación con métodos de optimización clásica, Mombello et al. (1996) proponen el método de LeverbergMarquard para estimar los parámetros del modelo del suelo; luego, mediante el cálculo de la resistencia de puesta a tierra equivalente, se corrigen los parámetros para que correspondan con distribución de potencial superficial del modelo del suelo. El método propuesto es válido para un número arbitrario de capas con valores arbitrarios de resistividad y es aplicable tanto a sistemas de puesta a tierra simples como complejos. Los resultados obtenidos fueron validados con el método de elementos finitos. Lagace et al. (2006) presentan un método para el cálculo de la resistividad aparente teórica mediante la generación de grupos de imágenes electrostáticas, y usando el método Marquard se realiza la estimación de los parámetros del suelo. Los errores máximos de los valores de resistividad calculados también se pueden estimar y se usan para validar el modelo de suelo.

Por otro lado, con métodos metaheurísticos, Gonos y Stathopulos (2005) y Zhiqiang y Bin (2011) proponen un algoritmo genético (GA, por sus siglas en inglés) para la estimación de los parámetros de un suelo de dos capas a partir de datos experimentales. El GA es comparado con el método de Seedher y Arora (1992), obteniendo resultados similares para un suelo de dos capas. También, Calixto et al. (2010) proponen un GA que genera los valores semillas de la población inicial usando el método de Pirson (Pirson, 1983). Los resultados presentados tienen desviaciones menores que los reportados por Gonos, aunque en algunos casos los resultados obtenidos no eran coherentes y no tenían ningún significado físico. He et al. (2013) usan el método de imágenes complejas y la expansión de Prony para el cálculo de la resistividad aparente teórica a partir de los parámetros de un suelo estratificado de forma horizontal. La función objetivo planteada corresponde a la raíz del error medio cuadrático entre los valores de resistividad aparente teóricos y medidos. He et al. (2013) plantean un algoritmo de optimización por enjambre de partículas (PSO, por sus siglas en inglés) para buscar los parámetros óptimos del modelo del suelo. 
En este artículo se presenta la implementación de una herramienta de optimización metaheurísticas novedosa denominada MVMO (por sus siglas en inglés) en la estimación de los parámetros del suelo de dos capas; se realizan comparaciones con otras herramientas metaheurísticas como GA, PSO y variantes como el Turbulent PSO (TPSO) y el Turbulent Crazy PSO (TCPSO). En ambas variantes se redefinen la forma en que se actualizan las velocidades de las partículas (Gutiérrez et al., 2017). La estructura del documento es la siguiente: en la segunda sección se describe la formulación matemática para el cálculo de la resistividad aparente usando el método de Wenner, y se plantea la función de optimización; en la tercera sección se describen los principales aspectos de la herramienta MVMO; en la cuarta sección se presentan algunos casos de estudios y se realizan análisis comparativos entre técnicas de optimización para solucionar el problema de estimación de parámetros. Finalmente, en la quinta sección se presentan las conclusiones.

\section{METODOLOGÍA}

En esta sección se presenta el método para medir la resistividad del suelo, el modelo del suelo adoptado a partir de las mediciones y la expresión matemática para el cálculo de los parámetros del suelo.

\section{Método de Wenner}

El método de Wenner es un arreglo tetraelectródico que se usa para medir la resistividad del suelo por medio de la ubicación de electrodos espaciados en línea recta, con una distancia $a$ entre cada par de electrodos, y enterrados a una profundidad tal que satisfaga la relación $a>20 b$, donde $b$ es la profundidad de enterramiento (IEEE, 2012; Moreno et al., 2007). En la figura 1 se muestra el arreglo del método de Wenner para la medición de la resistividad del suelo.

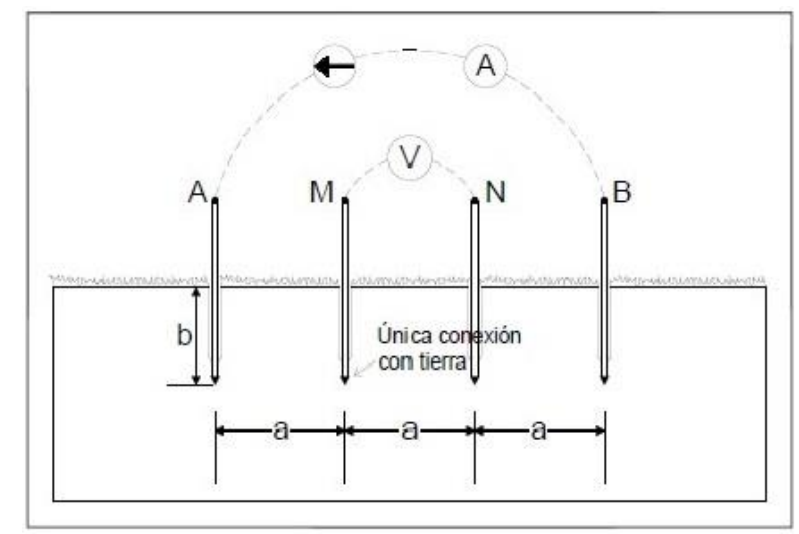

Fig. 1: Arreglo del método de Wenner

Mediante un telurómetro se hace circular una corriente $I$ entre los dos electrodos externos (electrodos de corriente) y se mide la caída de potencial $V$ con los dos electrodos internos (electrodos de potencial). El telurómetro realiza la relación $V / I$ dando un valor de resistencia aparente $R$ en ohmios. La resistividad aparente $\left(\rho_{a}\right)$ correspondiente a la resistividad medida aproximadamente a una profundidad $a$, se calcula considerando la resistencia entregada por el instrumento de medida mediante la ecuación (1).

$$
\rho_{a}=2 \pi \cdot a \cdot R
$$

\section{Suelo a dos capas horizontales}

El modelo del suelo se establece a partir de la interpretación de las mediciones de resistividad aparente obtenidas con el método de Wenner. El modelo de suelo a dos capas es a menudo una aproximación cercana a la condición real de suelo que consiste de dos capas horizontales homogéneas, como se observa en la figura 2. La primera, y más superficial, tiene una resistividad $\rho_{1}$ y su profundidad es $h$. La segunda, y más inferior, tiene una resistividad $\rho_{2}$ y una profundidad infinita. Este modelo tiene como principal ventaja que existen métodos para determinar de forma sencilla y precisa la resistencia de puesta a tierra y tensiones de toque y de paso, para diferentes configuraciones de sistemas de puestas a tierra (Heppe, 1979; Nahman y Amon, 1984).

\section{Estimación de los parámetros del modelo de dos capas}

Los métodos de modelamiento del suelo estratificado se clasifican en métodos de solución directa o indirecta, como lo han propuesto Oliveira et al. (2014). Los métodos de solución directa utilizan el algoritmo de Sunde (Sunde, 1949). 


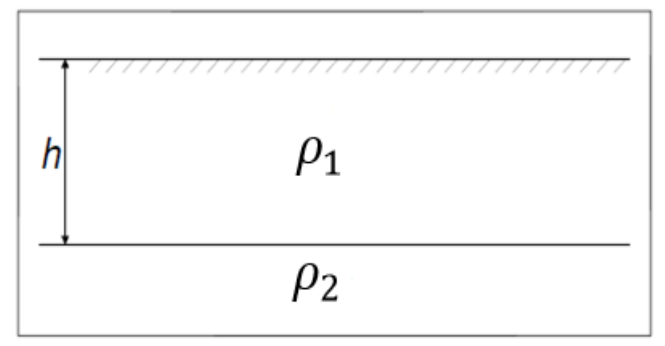

Fig.2: Suelo estratificado de dos capas

Este algoritmo está sujeto a que el calculista elija por inspección visual los valores de $\rho_{1}$ y $\rho_{2}$ de la gráfica de resistividad aparente obtenida por el método de Wenner, por lo que es posible obtener diferentes estructuras de dos capas para un mismo grupo de mediciones. Por otro lado, los métodos de solución indirecta utilizan métodos para la generación aleatoria de parámetros del suelo $\left(\rho_{i}, h\right)$ y de esta manera se generan también curvas teóricas que se calculan mediante la ecuación (2) (Tagg, 1964):

$$
\rho_{a} T(a)=\rho_{1}\left[1+4 \sum_{n=1}^{n=\infty}\left[\frac{k^{n}}{\left[1+\left(2 n \frac{h}{a}\right)^{2}\right]^{1 / 2}}-\frac{k^{n}}{\left[4+\left(2 n \frac{h}{a}\right)^{2}\right]^{1 / 2}}\right]\right]
$$

Donde la ec. (2.2) es el coeficiente de reflexión, $\rho_{1}$ la resistividad de la capa superior en $(\Omega . m), \rho_{1}$ la resistividad de la capa inferior en $(\Omega . \mathrm{m})$ y $h$ la profundidad de la capa superior.

$$
k=\frac{\rho_{2}-\rho_{1}}{\rho_{2}+\rho_{1}}
$$

Las curvas de resistividad teórica generadas son comparadas, numéricamente, con la experimental para cada conjunto de posibles soluciones de $\rho_{1}, \rho_{2}$ y $h$ de manera que se encuentren los parámetros asociados a un modelo que reproduzca de la mejor manera la misma curva experimental.

\section{Estimación de los parámetros del suelo}

La estimación de los parámetros del modelo de dos capas del suelo se establece como un problema de optimización no lineal con restricciones y se emplea el método indirecto para el cálculo de la resistividad aparente teórica a partir de los parámetros del suelo. Después, se compara con la curva de resistividad experimental. La función objetivo $(F O)$ planteada corresponde al error medio cuadrático entre los valores de resistividad aparente experimentales y teóricos, como se muestra en la ecuación (3). El planteamiento del problema de optimización es:

Minimizar:

$$
F O=\sum_{j=1}^{M}\left[\frac{\rho_{a_{j}}^{m}-\rho_{a_{j}}^{t}}{\rho_{a_{j}}^{m}}\right]^{2}
$$

Sujeto a:

$$
\begin{aligned}
& \rho_{1 \min } \leq \rho_{1} \leq \rho_{1 \max } \\
& \rho_{2 \min } \leq \rho_{2} \leq \rho_{2 \max } \\
& h_{1 \min } \leq h_{1} \leq h_{1 \max }
\end{aligned}
$$

Donde $\rho_{a j}{ }^{m}$, es la j-ésima medida de resistividad aparente del suelo con una distancia entre los electrodos de $a_{j}$ y $\rho_{a_{j}}^{t}$ es la resistividad aparente teórica con los parámetros $\rho_{1}, \rho_{2}$ y $h$ para la misma distancia. M es el número de medidas de resistividad realizadas. Las ecuaciones (4) a (6) son las restricciones del problema de optimización que corresponden a los límites superiores e inferiores para cada uno de los parámetros del modelo del suelo. 


\section{Algoritmo de optimización de mapeo media-varianza}

EI MVMO es un nuevo algoritmo de optimización heurística. Las técnicas heurísticas son técnicas de búsqueda basadas en un procedimiento inteligente que permite realizar optimización. Dicho proceso de optimización no es realizado con un proceso matemático riguroso. El MVMO fue concebido y desarrollado por István Erlich en 2010 (Erlich et al., 2010). El concepto básico comparte algunas similitudes con otras técnicas heurísticas, pero la característica novedosa es el uso de una función de mapeo aplicada para la mutación de las nuevas generaciones con base en la media y la varianza de la mejor población encontrada hasta el momento. La forma y localización de la curva de mapeo son ajustadas de acuerdo con el progreso de los procesos de búsqueda, y el MVMO actualiza el candidato de solución alrededor de la mejor solución en cada iteración. La variante enjambre de este algoritmo, $\mathrm{MVMO}^{\mathrm{s}}$, combina las bondades de la función de mapeo con la inteligencia de enjambre, lo cual vuelve el algoritmo más robusto frente a problemas de estancamiento en mínimos locales dentro del espacio de búsqueda (Erlich, 2018; Nakawiro et al., 2011).

El MVMOs opera sobre un conjunto de soluciones. Como en muchos algoritmos evolucionarios, su objetivo es ejecutar una optimización acertada y precisa con una mínima cantidad de funciones objetivos, evaluadas. El espacio de búsqueda interna de todas las variables en el MVMO está restringido a [0,1]. Así, los límites mínimos y máximos de las variables tienen que ser normalizados de 0 a 1 . Durante cada iteración no es posible que ningún componente del vector de solución viole los límites correspondientes. Para lograr este objetivo se desarrolló una función de mapeo especial. Las entradas de esta función son la media y varianza de las mejores soluciones que el MVMO ha descubierto hasta el momento. La salida de esta función de mapeo siempre está en el rango [0,1]. Esto quiere decir que la violación de los límites de variables durante el proceso de búsqueda no puede ocurrir. La forma y ubicación de la curva de mapeo son ajustadas de acuerdo al progreso en el proceso de búsqueda y el MVMO actualiza la solución candidata alrededor de la mejor solución en cada paso de la iteración. Así mismo, el MVMO es capaz de buscar alrededor de la mejor solución local con una posibilidad pequeña de quedar atrapado en los óptimos locales. Esta característica fue mejorada con la estrategia para el manejo de cero varianza (Cepeda et al., 2012).

Evaluación de la aptitud y manejo de restricciones: para cada individuo, se realiza la prueba de bondad chicuadrado; se revisa la viabilidad de la solución y se asigna un valor de aptitud. Se considera que un individuo es mejor si su aptitud es menor. El esquema de penalización estática es usado para el manejo de las restricciones. Dado que las variables de control son autolimitadas, todas las variables dependientes son restringidas aplicando la función integrada de aptitud como se muestra en la ecuación (7).

$$
\min f^{\prime}=f+\sum_{i=1}^{n} \mathrm{v}_{i} \max \left[0, g_{i}\right]^{\beta}
$$

Donde $f$ es la función objetivo original, $n$ es el número de restricciones, $\beta$ es el orden del término de penalización (por ejemplo 1 o 2), vi es el coeficiente de penalización de la $i$-ésima restricción y $g$ representa la restricción de desigualdad. Vale la pena mencionar que otras técnicas de manejo de restricciones son también aplicables al MVMO (Erlich et al., 2010).

Criterios de parada: el proceso de búsqueda del MVMO finaliza una vez se alcance un número predeterminado de evaluaciones de aptitud.

Archivo de solución: el archivo de solución constituye el conocimiento base del algoritmo para guiar la dirección de la búsqueda. Por lo tanto, los $n$ mejores individuos que el MVMO ha encontrado hasta el momento son guardados en el archivo. El valor de aptitud para cada individuo también es guardado. Las siguientes reglas son ajustadas para comparar el individuo generado en cada iteración y las soluciones existentes archivadas con el fin de evitar la pérdida de buenas soluciones: (i) cualquier solución viable se prefiere a cualquier solución inviable, (ii) entre dos soluciones viables, se prefiere la que tenga mejor valor de aptitud, (iii) entre dos soluciones inviables, se prefiere la que tenga menor valor de aptitud, por ejemplo, menor restricción de violación. Se realiza la actualización sólo si el nuevo individuo es mejor que aquellos que están en el archivo. El tamaño del archivo es fijo para todo el proceso. Los individuos archivados son organizados dinámicamente de modo que el primero siempre sea el mejor. Las soluciones viables son ubicadas en la parte alta del archivo. Estas soluciones son organizadas de acuerdo con sus valores de aptitud. Las soluciones inviables son organizadas de acuerdo con su aptitud y ubicadas en la parte más baja del archivo. Una vez el archivo esté completo con $n$ soluciones viables, cualquier solución de candidato inviable no tiene oportunidad de ser guardada en el archivo.

Asignación de padres: la primera solución posicionada (mejor hasta el momento), denotada como xbest, es asignada como el padre. 
Selección de variable: las búsquedas del MVMO alrededor de la media son guardadas en el archivo para la mejor solución sólo en $m$ direcciones seleccionadas. Esto quiere decir que sólo estas dimensiones de la descendencia serán actualizadas mientras que las $D-m$ dimensiones restantes toman los valores correspondientes del xbest. En este trabajo se implementó una estrategia de selección secuencial aleatoria.

Mutación: para cada $m$ dimensión seleccionada, la mutación se usa para asignar un nuevo valor a una variable. El procedimiento es el siguiente: dado un número aleatorio uniforme $x_{i} \in[0,1]$ el nuevo valor de la $i$ ésima variable se determina mediante la ecuación (8).

$$
x_{i}=h_{x}+\left(1-h_{1}+h_{0}\right) x_{i}^{\prime}-h_{0}
$$

Donde $h_{x}, h_{1}$ y $h_{0}$ son los resultados de la función de transformación de mapeo basado en diferentes entradas, calculadas mediante la ecuación (9).

$$
h_{x}=h\left(u_{i}=x_{i}^{\prime}\right), h_{0}=h\left(u_{i}=0\right), h_{1}=h\left(u_{i}=1\right)
$$

La función de mapeo se parametriza mediante la ecuación (10).

$$
h\left(\bar{x}_{i}, s_{i 1}, s_{i 2}, u_{i}\right)=\bar{x}_{i}\left(1-e^{-u_{i} s_{i 1}}\right)+\left(1-\overline{x_{i}}\right) e^{-\left(1-u_{i}\right) s_{i 2}}
$$

Donde $s_{i 1}$ y $s_{i 2}$ son factores de forma que permiten desniveles asimétricos de la función de mapeo. El desnivel se calcula mediante la ecuación (11).

$$
s_{i}=-\ln \left(v_{i}\right) f_{s}
$$

Donde $f_{s}$ es un factor de escala que permite el control del proceso de búsqueda durante la iteración. Para más detalles sobre cómo ajustar los dos diferentes factores, el de forma y el de escala, revisar (Cepeda et al., 2012). EI MVMO se ha aplicado satisfactoriamente para la solución de diferentes problemas de optimización en sistemas potencia tales como: el despacho de potencia reactiva óptima (Nakawiro et al., 2011), la identificación de equivalentes dinámicos (Cepeda et al., 2012), ubicación óptima y sintonización coordinada de controladores de amortiguamiento (Rueda et al., 2012), el control óptimo en granjas eólicas (Erlich et al., 2011) e identificación de parámetros de modelo de demanda (González-Longatt et al.,2012).

\section{RESULTADOS}

Para probar la efectividad del método de optimización propuesto y comparar su desempeño frente a otros métodos alternativos de solución, se evaluaron 9 casos que corresponden a mediciones de resistividad aparente analizados por varios autores (Alves et al., 2016; Gonos y Stathopulos, 2005; He et al., 2013; Zhiqiang y Bin, 2011). Los nueve casos considerados de medidas de resistividad, corresponden a mediciones en suelos de diversas estructuras, lo que se observa como cambios de tendencia en las curvas de resistividad en función de la distancia. Además, se tienen diferentes separaciones entre los electrodos y diferente número de mediciones en el método de Wenner. Estas variaciones en las medidas de resistividad afectan la convergencia del proceso de estimación de los parámetros del suelo. En la tabla 1 se presentan las mediciones realizadas con el método de Wenner para cada caso. Para evaluar el desempeño del MVMO en la estimación de los parámetros del suelo de dos capas se realiza la comparación con otras metaheurísticas, tales como: el GA, el PSO,TPSO y TCPSO reportadas en Gutiérrez et al.( 2017). Se define el espacio de búsqueda para las resistividades de las capas superficial y profunda entre 0 a $1500(\Omega$.m) y la profundidad de la capa superior entre 0 a $10(\mathrm{~m})$. El tamaño de la población para GA, PSO, TPSO y TCPSO es de 30 individuos, el número de generaciones es de 300 y se realizaron 50 simulaciones para cada caso con cada una de las metaheurísticas. Para el MVMO se definen 10 partículas y se realizan 9000 evaluaciones por cada simulación. La mejor solución corresponde al menor error obtenido calculado con la expresión (3) y se calcula la desviación estándar $\sigma$ del error para cada metaheurística con el fin de evaluar la convergencia de cada técnica.

Los parámetros estimados para cada caso se muestran en las tablas 2 a 10. En la segunda columna de cada una de las tablas, a modo de comparación, se presentan los parámetros del suelo obtenidos por los autores que propusieron originalmente los casos y que utilizaron diferentes técnicas y metodologías para la estimación, a saber: para el caso 1 fueron determinados por Dawalibi y Blattner (1984) a través de una metodología analítica basada en el método del Steepest-Descent. Para los casos 2 a 6, Alamo (1991) utilizó una técnica de gradiente de segundo orden, y para los casos 7 a 9 los parámetros del suelo fueron obtenidos por Seedher y Arora (1992) a través de una técnica que usa expresiones finitas de las ecuaciones de Wenner para los cálculos del potencial en suelos de dos capas. 
Tabla 1: Mediciones de resistividad aparente para cada uno de los casos considerados

\begin{tabular}{|c|c|c|c|c|c|c|c|c|c|}
\hline Caso & Mediciones & 1 & 2 & 3 & 4 & 5 & 6 & 7 & 8 \\
\hline \multirow{2}{*}{1} & $a(m)$ & 2.5 & 5 & 7.5 & 10 & 12.5 & 15 & & \\
\hline & $\rho_{a}(\Omega . m)$ & 320 & 245 & 182 & 162 & 168 & 152 & & \\
\hline \multirow{2}{*}{2} & $a(m)$ & 1 & 1.5 & 2.5 & 3 & 5 & 10 & & \\
\hline & $\rho_{a}(\Omega . m)$ & 255 & 290 & 315 & 376 & 528 & 690 & & \\
\hline \multirow{2}{*}{3} & $a(m)$ & 0.5 & 1 & 1.5 & 2 & 2.5 & 3 & 4 & 5 \\
\hline & $\rho_{a}(\Omega . m)$ & 58.71 & 61.79 & 58.1 & 61 & 73.79 & 78 & 79.1 & 78.2 \\
\hline \multirow{2}{*}{4} & $a(m)$ & 2.5 & 5 & 7.5 & 10 & 12.5 & 15 & 20 & 25 \\
\hline & $\rho_{a}(\Omega . m)$ & 451.6 & 366.7 & 250.2 & 180 & 144.2 & 120 & 116 & 96.5 \\
\hline \multirow{2}{*}{5} & $a(m)$ & 1 & 2 & 3 & 4 & & & & \\
\hline & $\rho_{a}(\Omega . m)$ & 156.4 & 113.1 & 95.2 & 65.3 & & & & \\
\hline \multirow{2}{*}{6} & $a(m)$ & 1 & 2 & 4 & 10 & 20 & 40 & & \\
\hline & $\rho_{a}(\Omega . m)$ & 136 & 140 & 214 & 446 & 685 & 800 & & \\
\hline \multirow{2}{*}{7} & $a(m)$ & 1 & 2 & 3 & 4 & 5 & & & \\
\hline & $\rho_{a}(\Omega . m)$ & 693.7 & 251.6 & 84.56 & 37.64 & 25.32 & & & \\
\hline \multirow{2}{*}{8} & $a(m)$ & 2 & 4 & 6 & 8 & 10 & & & \\
\hline & $\rho_{a}(\Omega . m)$ & 123.3 & 190 & 258.9 & 320.3 & 374.1 & & & \\
\hline \multirow{2}{*}{9} & $a(m)$ & 2 & 4 & 6 & 8 & 10 & & & \\
\hline & $\rho_{a}(\Omega . m)$ & 102.3 & 113.1 & 129.8 & 147.5 & 164 & & & \\
\hline
\end{tabular}

Tabla 2: Parámetros del suelo obtenidos para el caso 1, se compara con Dawalibi y Blattner (1984)

\begin{tabular}{|c|c|c|c|c|c|c|}
\hline Caso 1 & $\begin{array}{c}\text { Dawalibi y } \\
\text { Blattner }\end{array}$ & MVMO & GA & PSO & TPSO & TCPSO \\
\hline$\rho_{1}(\Omega . m)$ & 383.4982 & 372.726 & 371.799 & 372.502 & 372.305 & 331.751 \\
\hline$\rho_{2}(\Omega . m)$ & 147.6571 & 145.258 & 145.022 & 145.204 & 174.150 & 157.291 \\
\hline$h(m)$ & 2.5626 & 2.690 & 2.707 & 2.694 & 3.586 & 2.132 \\
\hline Error & 0.0079 & 0.00753 & 0.00753 & 0.00753 & 0.01064 & 0.01014 \\
\hline$\sigma$ & - & $9.1121 \mathrm{E}-07$ & 0.00134 & 0.09397 & 0.14796 & 0.11881 \\
\hline
\end{tabular}

Tabla 3: Parámetros del suelo obtenidos para el caso 2, se compara con Alamo (1991)

\begin{tabular}{|c|c|c|c|c|c|c|}
\hline Caso 2 & Alamo & MVMO & GA & PSO & TPSO & TCPSO \\
\hline$\rho_{1}(\Omega . m)$ & 244.71 & 246.836 & 246.782 & 246.853 & 292.230 & 186.920 \\
\hline$\rho_{2}(\Omega . m)$ & 1023.116 & 1058.633 & 1055.013 & 1058.878 & 966.290 & 856.804 \\
\hline$h(m)$ & 2.048 & 2.139 & 2.134 & 2.140 & 2.689 & 1.021 \\
\hline Error & 0.011 & 0.01074 & 0.01074 & 0.01074 & 0.01132 & 0.01134 \\
\hline$\sigma$ & - & $4.9729 \mathrm{E}-09$ & 0.00053 & 0.11372 & 0.16693 & 0.17114 \\
\hline
\end{tabular}

Tabla 4: Parámetros del suelo obtenidos para el caso 3, se compara con Alamo (1991).

\begin{tabular}{|c|c|c|c|c|c|c|}
\hline Caso 3 & Alamo & MVMO & GA & PSO & TPSO & TCPSO \\
\hline$\rho_{1}(\Omega . m)$ & 57.204 & 57.344 & 57.670 & 57.344 & 56.293 & 64.412 \\
\hline$\rho_{2}(\Omega . m)$ & 94.607 & 96.719 & 88.515 & 96.781 & 133.179 & 215.333 \\
\hline$h(m)$ & 1.546 & 1.651 & 1.449 & 1.653 & 2.557 & 2.980 \\
\hline Error & 0.024 & 0.024 & 0.02618 & 0.02402 & 0.02716 & 0.03416 \\
\hline$\sigma$ & - & 0.00011 & 0.00421 & 0.12459 & 0.03305 & 0.03375 \\
\hline
\end{tabular}


Tabla 5: Parámetros del suelo obtenidos para el caso 4, se compara con Alamo (1991).

\begin{tabular}{|c|c|c|c|c|c|c|}
\hline Caso 4 & Alamo & MVMO & GA & PSO & TPSO & TCPSO \\
\hline$\rho_{1}(\Omega . m)$ & 491.026 & 494.879 & 495.760 & 494.879 & 473.798 & 540.879 \\
\hline$\rho_{2}(\Omega . m)$ & 92.92 & 93.660 & 93.835 & 93.660 & 76.070 & 151.768 \\
\hline$h(m)$ & 4.431 & 4.370 & 4.359 & 4.370 & 2.929 & 2.785 \\
\hline Error & 0.0112 & 0.01103 & 0.01104 & 0.01103 & 0.01916 & 0.02137 \\
\hline$\sigma$ & - & $4.6251 \mathrm{E}-10$ & 0.00129 & 0.40141 & 0.56656 & 0.33938 \\
\hline
\end{tabular}

Tabla 6: Parámetros del suelo obtenidos para el caso 5, se compara con Alamo (1991).

\begin{tabular}{|c|c|c|c|c|c|c|}
\hline Caso 5 & Alamo & MVMO & GA & PSO & TPSO & TCPSO \\
\hline$\rho_{1}(\Omega . m)$ & 164.35 & 160.803 & 160.082 & 156.761 & 90.552 & 249.970 \\
\hline$\rho_{2}(\Omega . m)$ & 39.88 & 34.108 & 29.526 & 26.780 & 13.547 & 20.217 \\
\hline$h(m)$ & 1.698 & 1.847 & 1.951 & 2.048 & 3.368 & 1.247 \\
\hline Error & 0.0107 & 0.01037 & 0.01064 & 0.01080 & 0.02394 & 0.01301 \\
\hline$\sigma$ & - & 0.00013 & 0.02362 & 0.14201 & 0.06636 & 0.09272 \\
\hline
\end{tabular}

Tabla 7 : Parámetros del suelo obtenidos para el caso 6, se compara con Alamo (1991).

\begin{tabular}{|c|c|c|c|c|c|c|}
\hline Caso 6 & Alamo & MVMO & GA & PSO & TPSO & TCPSO \\
\hline$\rho_{1}(\Omega . m)$ & 122.379 & 125.528 & 125.374 & 125.500 & 180.437 & 113.992 \\
\hline$\rho_{2}(\Omega . m)$ & 1035.78 & 1093.298 & 1093.468 & 1093.027 & 1170.247 & 795.227 \\
\hline$h(m)$ & 2.465 & 2.713 & 2.711 & 2.711 & 3.813 & 2.397 \\
\hline Error & 0.0206 & 0.01698 & 0.01698 & 0.01698 & 0.02026 & 0.03283 \\
\hline$\sigma$ & - & $5.6783 \mathrm{E}-11$ & 0.00094 & 0.31161 & 0.32733 & 0.29646 \\
\hline
\end{tabular}

Tabla 8: Parámetros del suelo obtenidos para el caso 7, se compara con Seedher y Arora (1992).

\begin{tabular}{|c|c|c|c|c|c|c|}
\hline Caso 7 & $\begin{array}{c}\text { Seedhery } \\
\text { Arora }\end{array}$ & MVMO & GA & PSO & TPSO & TCPSO \\
\hline$\rho_{1}(\Omega . m)$ & 1003.35 & 999.659 & 996.970 & 999.641 & 948.525 & 711.782 \\
\hline$\rho_{2}(\Omega . m)$ & 21.14 & 19.985 & 19.944 & 19.984 & 5.272 & 50.901 \\
\hline$h(m)$ & 0.99 & 1.000 & 1.001 & 1.000 & 0.200 & 0.920 \\
\hline Error & 0.002 & $1.3419 \mathrm{E}-08$ & $5.0654 \mathrm{E}-06$ & $1.4229 \mathrm{E}-08$ & $1.9423 \mathrm{E}-04$ & $9.8735 \mathrm{E}-02$ \\
\hline$\sigma$ & - & 0.00110 & 0.03845 & 0.96518 & 0.67052 & 0.44928 \\
\hline
\end{tabular}

Tabla 9: Parámetros del suelo obtenidos para el caso 8, se compara con Seedher y Arora (1992).

\begin{tabular}{|c|c|c|c|c|c|c|}
\hline Caso 8 & $\begin{array}{c}\text { Seedhery } \\
\text { Arora }\end{array}$ & MVMO & GA & PSO & TPSO & TCPSO \\
\hline$\rho_{1}(\Omega . m)$ & 98.38 & 99.962 & 99.971 & 100.872 & 157.377 & 97.354 \\
\hline$\rho_{2}(\Omega . m)$ & 1018.8 & 998.367 & 991.377 & 1021.436 & 1379.429 & 936.573 \\
\hline$h(m)$ & 2.44 & 2.498 & 2.493 & 2.541 & 5.025 & 1.702 \\
\hline Error & 0.00039 & $5.4710 \mathrm{E}-08$ & $1.4137 \mathrm{E}-06$ & $1.2512 \mathrm{E}-05$ & 0.00181 & 0.00205 \\
\hline$\sigma$ & - & $3.2512 \mathrm{E}-05$ & 0.00081 & 0.11664 & 0.20682 & 0.22442 \\
\hline
\end{tabular}


Tabla 10: Parámetros del suelo obtenidos para el caso 9, se compara con Seedher y Arora (1992).

\begin{tabular}{|c|c|c|c|c|c|c|}
\hline Caso 9 & $\begin{array}{c}\text { Seedhery } \\
\text { Arora }\end{array}$ & MVMO & GA & PSO & TPSO & TCPSO \\
\hline$\rho_{1}(\Omega . m)$ & 99.99 & 99.997 & 99.943 & 99.833 & 74.506 & 120.656 \\
\hline$\rho_{2}(\Omega . m)$ & 302.64 & 299.722 & 306.713 & 292.208 & 316.465 & 302.796 \\
\hline$h(m)$ & 5.04 & 4.997 & 5.063 & 4.904 & 5.202 & 4.849 \\
\hline Error & $6.8019 \mathrm{E}-06$ & $7.7625 \mathrm{E}-09$ & $8.1565 \mathrm{E}-06$ & $6.4386 \mathrm{E}-06$ & $4.7801 \mathrm{E}-05$ & $2.2503 \mathrm{E}-09$ \\
\hline$\sigma$ & - & $5.6243 \mathrm{E}-05$ & 0.00048 & 0.03526 & 0.03326 & 0.03401 \\
\hline
\end{tabular}

Con el GA se obtuvieron errores pequeños; aunque para los casos 3 y 9 los errores obtenidos son superiores a los obtenidos por Alamo (1991) y por Seedher y Arora (1992), respectivamente. Por su parte, con el PSO sólo para el caso 5, se obtuvo un error superior al obtenido por Alamo. Por otro lado, las técnicas TPSO y TCPSO fueron las de peor desempeño, aunque se tienen resultados que podrían considerarse buenos, el error obtenido es superior al reportado por los autores que proponen los casos. Para el caso 7, todas las técnicas entregaron parámetros del suelo con errores menores a los presentados por los autores, excepto el TCPSO. Mientras que para el caso 3, sólo el MVMO igualó el error presentado por Alamo (1991). El resto superan el error presentado por los autores.

Para analizar la estabilidad en la convergencia de las soluciones entregadas por el MVMO, de las 50 corridas realizadas para cada caso de suelo, se calcularon los valores mínimos, máximo, promedio y desviación estándar del error y los parámetros del suelo. En la tabla 11 se muestran los anteriores cálculos para el caso 3 , que se considera, de los simulados, el más complejo. En la tabla 11 se puede observar que los parámetros obtenidos por el MVMO presentan una desviación reducida comparado con la media; por ejemplo, para los parámetros estimados la mayor desviación estándar corresponde al $2.43 \%$ del valor de la media.

Tabla 11: Análisis de resultados de las 50 corridas para el caso 3 con MVMO

\begin{tabular}{|c|c|c|c|c|}
\hline Caso 3 & Error & $\rho_{1}(\Omega . m)$ & $\rho_{2}(\Omega . m)$ & $h(m)$ \\
\hline $\min$ & 0.02402 & 57.34435 & 96.69909 & 1.65142 \\
\hline max & 0.02476 & 58.12874 & 103.63164 & 1.91619 \\
\hline prom & 0.02404 & 57.38183 & 97.02763 & 1.66381 \\
\hline Desv est & 0.00011 & 0.11599 & 1.05602 & 0.04041 \\
\hline
\end{tabular}

Utilizando la expresión (2) y los parámetros del suelo obtenidos, se calculan los valores de resistividad aparente teóricos para diferentes valores de separación entre electrodos a. En las figuras 3 (a) y 3 (b) se presentan los valores de resistividad aparente teóricos correspondientes a los casos 3 y 7 respectivamente. Además, en las figuras se superponen los valores de resistividad medidos y la resistividad calculada para los parámetros obtenidos por Alamo (1991), Seedher y Arora (1992) y los parámetros obtenidos con MVMO.

En la figura 3 (a), para el caso 3, se observa que el MVMO se ajusta de forma aceptable a la característica de resistividad del suelo medida y es coincidente con los resultados presentados por Alamo (1991). Analizando el perfil de resistividad de este caso se concluye que presenta complejidad, ya que a partir del análisis de los puntos de inflexión se determina que es un suelo de más de dos capas. Para el caso 7 , presentado en la figura 3 (b), se observa que el ajuste del modelo obtenido es mejor y prácticamente no hay diferencia en los perfiles calculados con los parámetros reportados por Seedher y Arora (1992) y los obtenidos con MVMO.

En las figuras 4 (a) y 4 (b) se observa el proceso de convergencia para cada técnica de optimización en una corrida para estimación de los parámetros del suelo del caso 3. De la figura 4 (a) se aclara que cada iteración corresponde a la evaluación de 30 individuos o partículas en el GA, PSO, TPSO y TCPSO, y para MVMO cada evaluación corresponde a la valoración de una partícula (ver figura 4 (b)). Realizar una comparación en la convergencia de las metaheurísticas implica que para las 300 iteraciones mostradas en la figura 4 (a) se deben realizar 9000 evaluaciones en MVMO, y cada técnica de optimización tiene el mismo número de evaluaciones. Observando las figuras 4 (a) y 4 (b), se concluye que es mayor la velocidad de convergencia del MVMO. Una diferencia es que el MVMO escoge su población inicial de forma aleatoria inicial con valor alto de la función objetivo, la cual se reduce muy rápidamente, como se muestra en la figura 4 (b). 


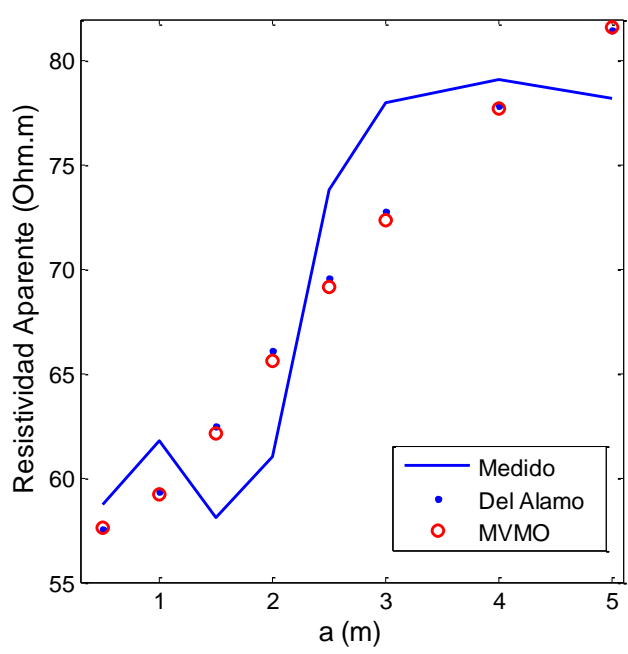

(a)

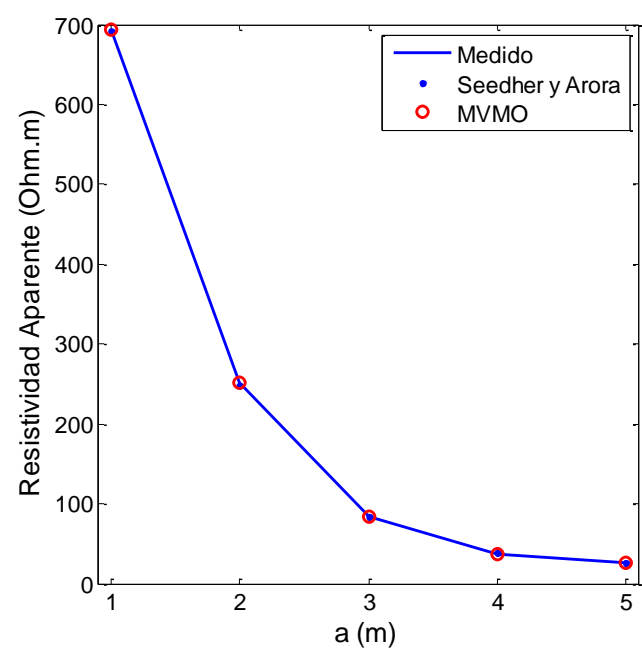

(b)

Fig. 3: (a) Comparación entre la resistividad aparente medida y teórica obtenida con los parámetros de Alamo (1991) y MVMO para el caso 3. (b) Comparación entre la resistividad aparente medida y teórica obtenida con los parámetros de Seedher y Arora (1992) y MVMO para el caso 7

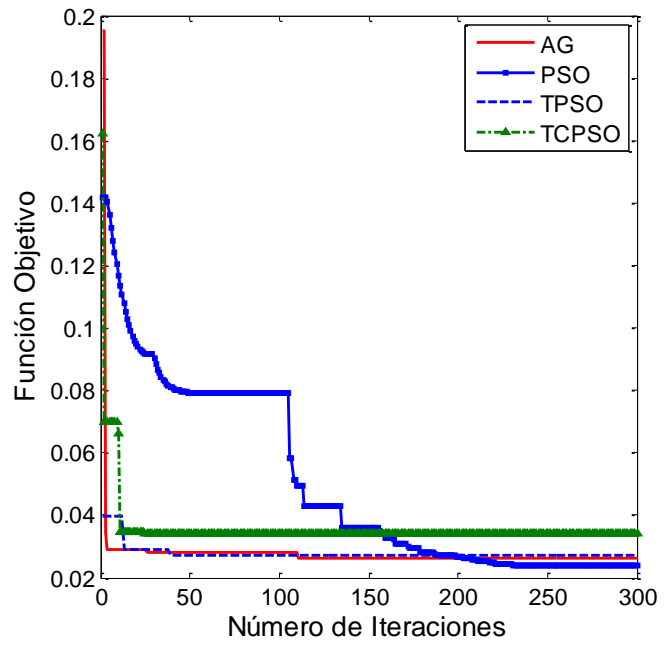

(a)

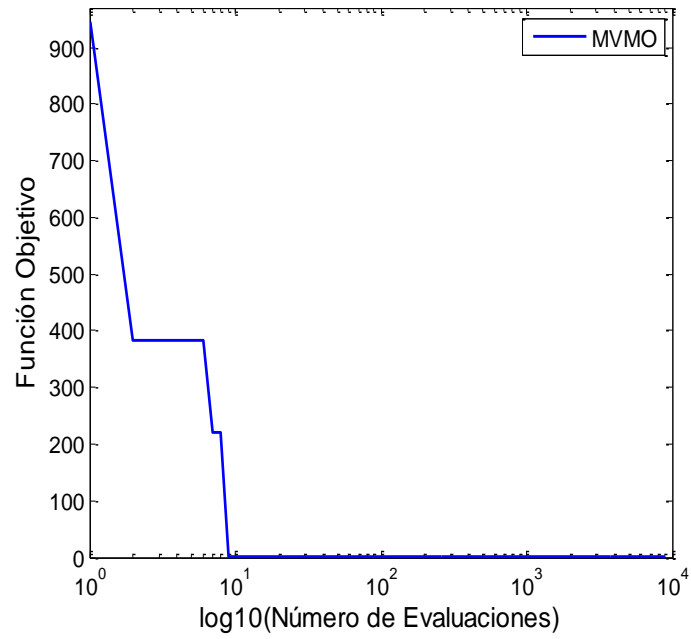

(b)

Fig. 4: (a) Convergencia para el caso 3 con GA, PSO, TPSO y TCPSO. (b) Convergencia del MVMO a través de las evaluaciones para el caso 3

\section{CONCLUSIONES}

En este artículo se presentó la estimación de los parámetros del suelo de dos capas solucionado mediante la técnica de optimización MVMO. La estimación de los parámetros del suelo es un problema que presenta no linealidad, no convexidad y múltiples óptimos locales. La técnica propuesta de MVMO se comparó con GA, PSO y sus variantes TPSO y TCPSO. Los resultados para los casos 1 a 9 evidenciaron la aplicabilidad y efectividad de la técnica MVMO.

La comparación del MVMO con las técnicas de optimización implementadas y las reportadas en la literatura técnica permite concluir que el MVMO resulta ser mucho más competitivo que el GA, PSO convencional y sus variantes, e incluso se obtienen soluciones de mejor calidad que las reportadas con las técnicas de optimización clásica. Esto se debe a las características del MVMO de localización y forma de función de mapeo y la estrategia de actualización del candidato. Esto hace que la exploración del espacio de búsqueda sea más efectiva, lo que permite al algoritmo escapar de óptimos locales.

\section{REFERENCIAS}

Alamo, J. L., A Comparison among Eight Different Techniques to Achieve an Optimum Estimation of Electrical Grounding Parameters in Two-Layered Earth, doi:10.1109/61.248299, IEEE Transactions on Power Delivery, 8(4), 1890-1899 (1993) 
Alamo, J. L., A Second Order Gradient Technique for an Improved Estimation of Soil Parameters in a Two-Layer Earth, doi:10.1109/61.85863, IEEE Transactions on Power Delivery, 6(3), 1166-1170 (1991)

Alves, A.C.B., R. P. Marinho, G. A. A. Brigatto y L.P. Garces, Multilayer Stratification Earth by Kernel Function and QuasiNewton Method, doi:10.1109/TLA.2016.7430083, IEEE Latin America Transactions, 14(1), 225-234 (2016)

Calixto, W.P., L.M. Neto y otros tres autores, Parameters Estimation of a Horizontal Multilayer Soil using Genetic Algorithm, doi: 10.1109/TPWRD.2010.2040845, IEEE Transactions on Power Delivery, 25(3), 1250-1257 (2010)

Cepeda, J. C., J. L. Rueda e I. Erlich, Identification of Dynamic Equivalents based on Heuristic Optimization for Smart Grid Applications, IEEE Congress on Evolutionary Computation, 1-8, Brisbane-Australia, 10-15 de Junio (2012)

Dawalibi, F. y C. J. Blattner, Earth Resistivity Measurement Interpretation Techniques, doi:10.1109/TPAS.1984.318254, IEEE Transactions on Power Apparatus and Systems, PAS-103(2), 374-382 (1984)

Erlich, I., Mean-Variance Mapping Optimization Algorithm home page (2018)

Erlich, I., W. Nakawiro y M. Martínez, Optimal Dispatch of Reactive Sources in Wind Farms, doi:10.1109/PES.2011.6039534, IEEE Power and Energy Society General Meeting, 1-7 (2011)

Erlich, I., G. K. Venayagamoorthy y N. Worawat, A Mean-Variance Optimization Algorithm, IEEE Congress on Evolutionary Computation, 1-6, Barcelona-España, 18-23 de Julio (2010)

Gonos, I.F. e I.A. Stathopulos, Estimation of Multilayer Soil Parameters using Genetic Algorithms, doi:10.1109/TPWRD.2004.836833, IEEE Transactions on Power Delivery, 20(1), 100-106 (2005)

Gonzalez-Longatt, F., J. Rueda y otros tres autores, Identification of Gaussian Mixture Model using Mean Variance Mapping Optimization: Venezuelan Case, $3^{\text {rd }}$ IEEE PES Innovative Smart Grid Technologies (ISGT) Europe Conference, 1 6, Berlín-Alemania, 14-17 de Octubre (2012)

Gutiérrez, D., W. M. Villa y J. M. López-Lezama, Flujo Óptimo Reactivo mediante Optimización por Enjambre de Partículas, doi: 10.4067/S0718-07642017000500020, Información Tecnológica, 28(5), 215-224 (2017)

He, W., R. Zhang y otros cuatro autores, Parameter Estimation of Horizontal Multilayer Earth based on Complex Image Method and Improved Particle Swarm Optimization, doi:10.1002/tee.21880, IEEE Transactions on Electrical and Electronic Engineering, 8(5), 456-462 (2013)

Heppe, A. J., Computation of Potential at Surface Above an Energized Grid or Other Electrode Allowing for Non-Uniform Current Distribution, doi:10.1109/TPAS.1979.319377, IEEE Transactions on Power Apparatus and Systems, PAS-98(6), 1978-1989 (1979)

IEEE Std 81, Guide for Measuring Earth Resistivity, Ground Impedance, and Earth Surface Potentials of a Grounding System, 1-86, Nueva York, Estados Unidos (2012)

IEEE Std 80, Guide for Safety in AC Substation Grounding, 1-226, Nueva York, Estados Unidos (2015)

Lagace, P.J., M.H. Vuong, M. Lefebvre y J. Fortin, Multilayer Resistivity Interpretation and Error Estimation using Electrostatic Images, doi: 10.1109/TPWRD.2006.874618, IEEE Transactions on Power Delivery, 21(4), 1954-1960 (2006)

Mombello, E., O. Trad, J. Rivera y A. Andreoni, Two-layer Soil Model for Power Station Grounding System Calculation considering Multilayer Soil Stratification, doi:10.1016/0378-7796(96)01042-5, Electric Power Systems Research, 37, $67-78$ (1996)

Moreno, G. M., J. A. Valencia, C. A. Cárdenas y W. M. Villa, Fundamentos e Ingeniería de las Puestas a Tierra: Respuestas ante Fallas Eléctricas y Rayos, $1^{\text {a }}$ Ed., 1-192, Universidad de Antioquia, Medellín, Colombia (2007)

Nahman, J. y D. S. Amon, Analytical Expressions for the Resistance of Grounding Grids in Nonuniform Soil, doi:10.1109/TPAS.1984.318367, IEEE Transactions on Power Apparatus and Systems, PAS-103(4), 880-885 (1984)

Nakawiro, W., I. Erlich y J. L. Rueda, A Novel Optimization Algorithm for Optimal Reactive Power Dispatch: A Comparative Study, 4th International Conference on Electric Utility Deregulation and Restructuring and Power Technologies (DRPT), 1555-1561, Shandong-China, 6-9 de Julio (2011)

Oliveira, D., W.P. Calixto y otros tres autores, Multilayer Soil Parameters Estimation Optimization Using Genetic Algorithms, International Conference on Grounding and Earthing and $6^{\text {th }}$ International conference on Lighting Physics and Effects, Manaus - Brasil, Mayo (2014)

Pirson, S. J., Geologic Well Log Analysis, 3a Ed., 496. Gulf Publishing Co, Houston, Estados Unidos (1983)

Rueda, J. L., J. C. Cepeda e I. Erlich, Estimation of Location and Coordinated Tuning of PSS based on Mean-Variance Mapping Optimization, doi:10.1109/PESGM.2012.6345025, IEEE Power and Energy Society General Meeting, 1-8 (2012)

Seedher, H.R. y J.K. Arora, Estimation of Two Layer Soil Parameters using Finite Wenner Resistivity Expressions, doi:10.1109/61.141833, IEEE Transactions on Power Delivery, 7(3), 1213-1217 (1992)

Sunde, E.D., Earth Conduction Effects in Transmission Systems, $1^{\text {a }}$ Ed., 1- 373. D. Van Nostrand Co, New York, United States (1949)

Tagg, G. F., Earth Resistances, $1^{a}$ Ed., 1-276, Pitman Publishing Corporation, New York, United States (1964)

Zhiqiang, H. y Z. Bin, Soil Model's Inversion Calculation based on Genetic Algorithm, doi:10.1109/APL.2011.6110113, 7th Asia-Pacific International Conference on Lightning, 225-230 (2011) 
